\title{
Electronic control of elastomeric microfluidic circuits with shape memory actuators $\dagger+$
}

\author{
Saurabh Vyawahare, ${ }^{* a, c}$ Suresh Sitaula, ${ }^{b}$ Sujitha Martin, ${ }^{b}$ Dvin Adalian ${ }^{b}$ and Axel Scherer ${ }^{a, c}$ \\ Received 17th March 2008, Accepted 3rd June 2008 \\ First published as an Advance Article on the web 9th July 2008 \\ DOI: 10.1039/b804515a
}

Recently, sophisticated fluidic circuits with hundreds of independent valves have been built by using multi-layer soft-lithography to mold elastomers. However, this shrinking of microfluidic circuits has not been matched by a corresponding miniaturization of the actuation and interfacing elements that control the circuits; while the fluidic circuits are small ( $\sim 10-100$ micron wide channels), the Medusa's head-like interface, consisting of external pneumatic solenoids and tubing or mechanical pins to control each independent valve, is larger by one to four orders of magnitude ( $\sim \mathrm{mm}$ to $\mathrm{cm}$ ). Consequently, the dream of using large scale integration in microfluidics for portable, high throughput applications has been stymied. By combining multi-layer soft-lithography with shape memory alloys (SMA), we demonstrate electronically activated microfluidic components such as valves, pumps, latches and multiplexers, that are assembled on printed circuit boards (PCBs). Thus, high density, electronically controlled microfluidic chips can be integrated alongside standard opto-electronic components on a PCB. Furthermore, we introduce the idea of microfluidic states, which are combinations of valve states, and analogous to instruction sets of integrated circuit (IC) microprocessors. Microfluidic states may be represented in hardware or software, and we propose a control architecture that results in logarithmic reduction of external control lines. These developments bring us closer to building microfluidic circuits that resemble electronic ICs both physically, as well as in their abstract model.

Marshall Mcluhan's “The medium is the message”, is a familiar refrain in communication media; its complement in technology - the idea that the platform chosen is as important as the individual components in determining the final impact, rings true for the electronics revolution, an impossibility without platforms like silicon wafers and printed circuit boards. Recently, in microfluidics, ${ }^{1}$ the creation of low-cost integrated fluidic circuits with soft elastomers (i.e. 'silicone instead of silicon') $)^{2-4}$ raised hopes that it is a similar platform for fluidic devices. However, in practice, the initial expectations have been tempered, because in highly integrated elastomeric chips, controlling each independent valve results in a morass of pneumatic control lines and unwieldy, expensive control systems. Consequently, other materials and forces to power micro-valves have been explored..$^{5-8}$ The most promising are electrical platforms like those made with Braille or dot matrix printer pins, ${ }^{9,10}$ conjugative

${ }^{a}$ Caltech Microfluidic Foundry, Kavli Nanoscience Institute, California Institute of Technology, 1200 E. California Blvd., Pasadena, CA, 91125, USA.E-mail: saurabhv@caltech.edu

${ }^{b}$ Summer Undergraduate Research Program, California Institute of Technology, 1200 E. California Blvd., Pasadena, CA, 91125, USA 'Division of Engineering and Applied Sciences, California Institute of Technology, 1200 E. California Blvd., Pasadena, CA, 91125, USA $\dagger$ Electronic supplementary information (ESI) available: Movie showing in real time a 76 micron diameter wire being actuated using a $240 \mathrm{~mA}$ current. It is easy to visually determine that the channel is completely squeezed shut by the wire on actuation. See DOI: $10.1039 / \mathrm{b} 804515 \mathrm{a}$ $\$$ The HTML version of this article has been enhanced with colour images. polymers ${ }^{11}$ paraffin wax ${ }^{12,13}$ and electro-rheological fluids. ${ }^{14}$ But, as yet, no single method can be considered ideal with regard to scalability, automation, control simplicity, cost and ease of integration with both opto-electronics and the sophisticated circuits made in elastomers.

Here, we introduce a platform on printed circuit boards (PCBs) that combines microfluidic circuits defined in elastomers with shape memory alloy (SMA) wires as the control element to address these problems. Elastomers are rubber-like polymers that can take large strains without failure, whereas shape memory alloys (SMAs) exert large stresses on undergoing the diffusionless, solid state, shape memory transition. ${ }^{15}$ SMAs are ideal for defining small actuators ${ }^{16,17}$ because they have a very large work to volume ratio $\left(\sim 10^{7} \mathrm{~J} \mathrm{~m}^{-3}\right)$. Elastomers provide electrical insulation, thermal insulation and a restoring force proportional to the distortion caused by the SMA. These orthogonal sets of properties allows an ideal marriage. Previously, this combination has been largely underappreciated, except in a few bio-medical applications that are limited in scope because they involve either single valves controlling single millimeter size tubes or SMA valves with a control interface as large as that for standard pneumatic valves. ${ }^{18-21}$ We show that the combination of multi-layer soft lithography with SMA wires on printed circuit board provides for a versatile platform allowing several types of microfluidic component designs.

We used poly-dimethyl siloxane (PDMS) for elastomer, and $\mathrm{Ni}-\mathrm{Ti}$ alloy wires for SMA material. ${ }^{22}$ PDMS is transparent, inexpensive and compatible with most aqueous solutions. For applications involving organic solvents, fluorinated 
elastomers ${ }^{23,24}$ can replace PDMS. Ni-Ti alloys are currently the most commonly used shape memory alloys. Besides Ni-Ti alloys, there are a large variety of SMA materials available with a wide range of properties to choose from. ${ }^{25}$ Mechanically drawn wire forms of SMAs ("muscle wires"), where grains are aligned for maximum elongation, are available at low cost $(\sim$ few dollars per meter) and have reliability for millions of actuation cycles. ${ }^{22}$

The basic valve design involves looping a SMA wire (typically, $0.003^{\prime \prime}$ in diameter) around a fluidic channel (typically 100 microns wide, 10 microns high channel with a rounded profile) defined within elastomer. The SMA wire passes through punched holes and through vias on a rigid PCB board and is soldered down at both ends to facilitate electrical contact. After soldering, embedding the wire completely within PDMS increases the pressure that the elastomeric chip can withstand before delaminating and increases fabrication tolerance for the wire tension. If the wire is too tense, the channel is closed before any actuation; if too slack, the forces may not be directed to the valve region. Embedding the wire in PDMS could result in an unpredictable membrane thickness above the looped wire, but in practice, this procedure does not drastically change valve repeatability. On conducting a current through the wire, resistive heating raises the temperature of the wire to transition temperature, shortening the wire's length, and squeezing the channel shut (Fig. 1).

A single wire can close multiple adjacent channels simultaneously (Fig. 2b). This parallel operation saves space and power in high density chips. We may want to use a single SMA wire to close some microfluidic channels while leaving adjacent channels unchanged-like the pneumatic cross-over geometry. This is accomplished by using a 'block' or 'plunger' above the channels we desire to close (Fig. 2a), made in elastomer or more rigid material. If an elastomer block is used, another layer of PDMS is bonded to the chip so that the protrusions/blocks in this layer align with the channels that need to have a valve. The 'plunger' must be smaller than the channel width and tall enough that the wire can push down on it to close the channel. Fluidic system design without plungers involves no elastomer layer alignment and withstands larger pressures, whereas the design with plungers can be employed for building combinatorial multiplexers $^{26}$ (Fig. 2d and e), but needs accurate alignment in fabrication.

The first generation design with 76 micron diameter SMA wires required $\sim 50 \mathrm{~mA}$ (air cooled-plunger design) to $\sim 230 \mathrm{~mA}$ (embedded in elastomer) current in the on state, with power requirements $<0.5 \mathrm{~W}$ for each valve. Due to hysteresis, it is possible to actuate a wire at a certain current and then lower it, while keeping the wire contracted, reducing power consumption by as much as $60 \%$ compared to the initial power. The valve actuates at seconds to sub-second speeds with the heat transfer and dynamic response of elastomer being the determining factor (see ESI video and Fig. 1e and $\mathrm{f}^{\dagger}$ ). In our designs, the SMA micro-valves were able to withstand a pressure differential of 5-15 psi, with failure usually occurring through de-lamination of bonded elastomer layers, unrelated to SMA wires. Plasma bonding of PDMS layers, instead of the thermal bonding that we used, may help in sustaining higher pressures. A valve was tested for over 10000 cycles and suffered no significant change in performance.
SMA valves do involve some trade-offs. Intrinsically, the shape memory transformation is nano-seconds fast, but the thermal transfer rates limit the on-to-off rate (the off-to-on rate can be increased almost arbitrarily by pulsing high current ${ }^{27}$ ). So a peristaltic pump (Fig 2c) can only cycle at 1-2 Hz. Active cooling schemes like Peltier cooling or liquid cooling (particularly suitable for microfluidic devices) can increase speed. Choices involving wire dimensions and properties also help - wires with higher transition temperature cool faster, but need greater power to reach that transition temperature. Smaller diameter wires cool faster, having better surface/volume ratio and use lower power (scaling as $r^{2}$ ) due to greater resistance. Thus, wire miniaturization increases speed and reduces required power, but multiple small diameter wires may be needed to generate larger forces. The best cooling time constants, without active cooling, reported in the literature are the order of $0.1 \mathrm{~s}^{27}$

Another inter-related issue with SMA valves is that heat can be transmitted to the channel contents if the wire is heated (contracted) for several minutes. This heat dissipation problem can be surmounted by utilizing smaller diameter SMA wires, employing better heat management or using wires with lower transition temperature. However, a fourth approach has many advantages; it involves arranging SMA valves to control pressure to pneumatic valves, that in turn regulate the fluid flow. The wire is now located upstream or downstream of a membrane valve and controls a pressurized control line (Fig. 3). Both normally open and normally closed valves can be made adding to the versatility of this design.

We can construct a pressure latch-or pressure storage unit as shown in Fig. 3e. Instead of air, a non-volatile liquid is used. We were able to keep the valve in a closed state without applied pressure for over one hour at which point the experiment was stopped. The control over the pneumatic manifold system also enables the construction of control multiplexers as described earlier, using the plunger design. Thus, a set of valves or a set of flow lines can be multiplexed. Note that one pressure supply (possibly from a small motor or infusion pump) is sufficient for all valves on the chip - as opposed to having a solenoid and tubing for each valve. Indirect valves separate out the SMA valves and the fluidic circuit, and each can be built separately without interfering with the design of the other, allowing for higher density circuits.

Being electrically powered allows SMA valves to have a smaller footprint compared with pneumatic solenoids and tubes needed from pneumatic valves. The main advantage of the direct valve is that it is simpler to create, whereas the indirect valve is more versatile. In our experiments, we used only the commercially made $70{ }^{\circ} \mathrm{C}$ transition temperature SMA wires, which can cause heat transfer problems in direct valves, if valves are actuated for a long time. However, SMA materials can be manufactured with a large range of transition temperatures and economics will dictate the type of design used. We believe both designs compare favorably with plain pneumatic designs for several applications.

\section{Control architectures}

Before describing control architectures, ${ }^{28}$ it is useful to consider an abstract microfluidic chip with $N$ two-state valves. At any 
a

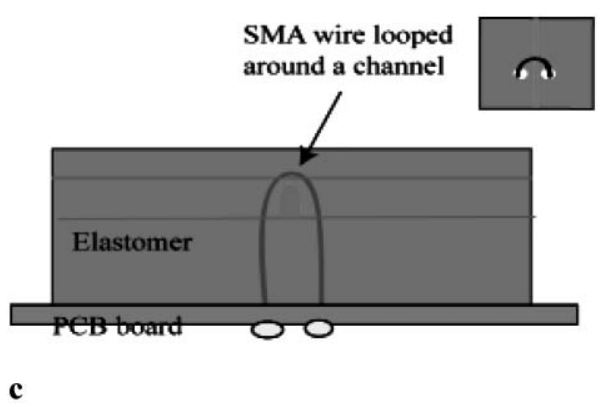

b
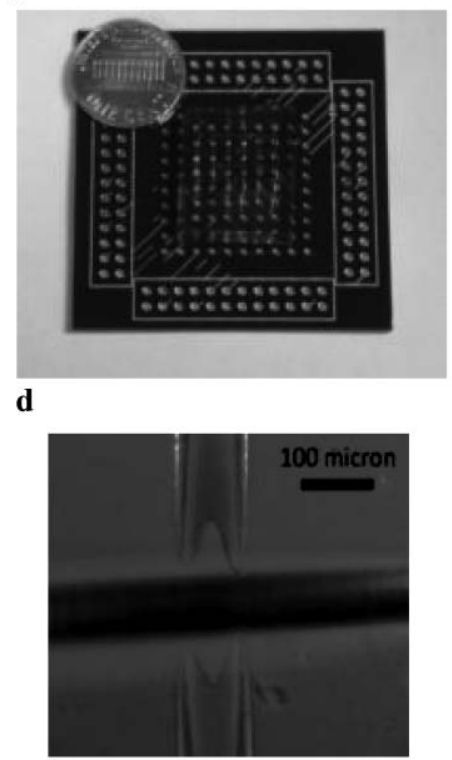

$\mathbf{e}$

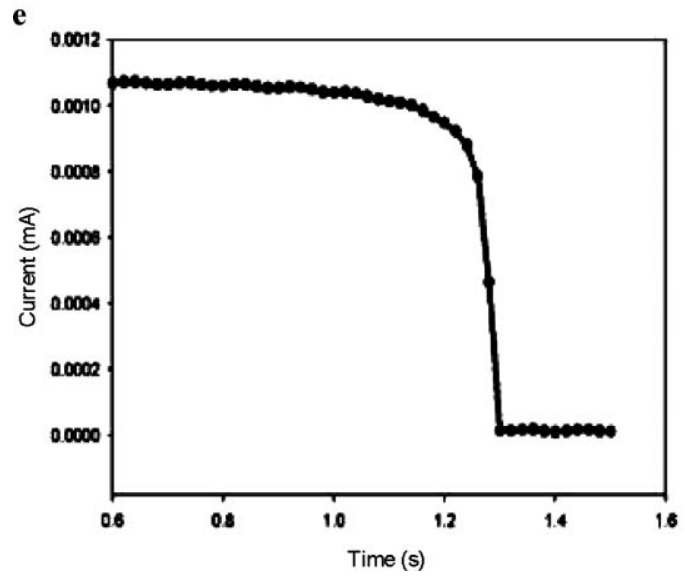

f

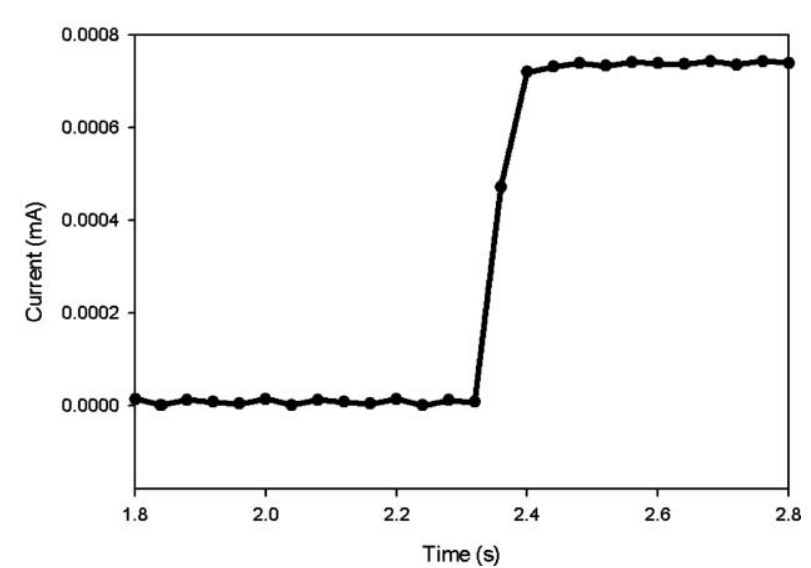

Fig. 1 Design of direct SMA valves. (a) Design of an SMA valve. An SMA wire is looped around a rounded channel, soldered to a PCB board and embedded in PDMS (not to scale). (b) A PDMS chip with several valves on a PCB board. (c) Single wire, embedded valve with 76 micron diameter wire on top of a 100 micron wide and 13 micron tall, rounded channel. (d) On resistively heating the SMA wire, it contracts and squeezes the channel shut. (e) Typical off-to-on response of an SMA wire. A $230 \mathrm{~mA}$ current was used to turn on the wire at $t=0 \mathrm{~s}$. Channel width $=120 \mu \mathrm{m}$ and pressure $=5.0 \mathrm{psi}$. An AC current through a solution of $\mathrm{KCl}$ inside the channel is monitored. The off-to-on rate speed can be arbitrarily increased by passing a higher current. (f) Typical on-to-off response. A $120 \mu \mathrm{m}$ channel pressurized to $10 \mathrm{psi}$ is closed for $10 \mathrm{~s}$ using $230 \mathrm{~mA}$ of current. Current is turned off at $t=0 \mathrm{~s}$. (Also see ESI video file. $\dagger$ ).

time, the chip is in a particular state, represented by a binary encoded number (e.g. 11101011, where 1 is the "open" and 0 the "closed" state, the position of the bit determining valve location). A chip is a particular type of a finite state machine, which cycles through various states to complete a task. What could a state be? A state could represent an instruction like 'open port A' or 'send contents of chamber A to chamber B' or 'mix contents of A and B'; in general, anything that can be done with a combination of valves. Each chip can only be in one state at a given time. Thus, each state of a microfluidic chip is analogous to the machine language instruction of a microprocessor, with one instruction/state running at a given time to a clock cycle. The total number of possible states is $2^{N}$.

Now, instead of actuating valves, we may consider ways to actuate a state, and this needs a way of actuating multiple valves at the same time. We present two architectures to control states: the first involves directly connecting valves and electrical control lines (Fig. 4a). Multiple wires may be arranged in addressable columns and rows to reduce control lines. ${ }^{29}$ Wires are actuated using constant current or voltage, with pulse width modulation (PWM $)^{30}$ on each line if tuning is required. Signals for a state can be programmed in software and sent out timed to a clock cycle by a microcontroller to a peripheral driver (Darlington or Mosfet array) with a current/voltage source to activate the corresponding wires. For a few wires/valves simple spatial multiplexing is sufficient. For multiple wires, time division multiplexing can lower the peak power with only one wire being activated in a time slot (see Methods).

The second architecture involves microfluidic states $(S)$ being represented in software or hardware (Fig. 4b). This representation of states can itself be controlled by a multiplexer (electronic or fluidic). To distinguish this architecture from others, we 
a

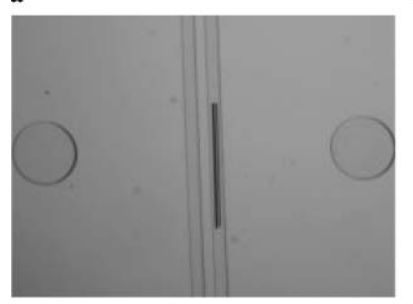

c
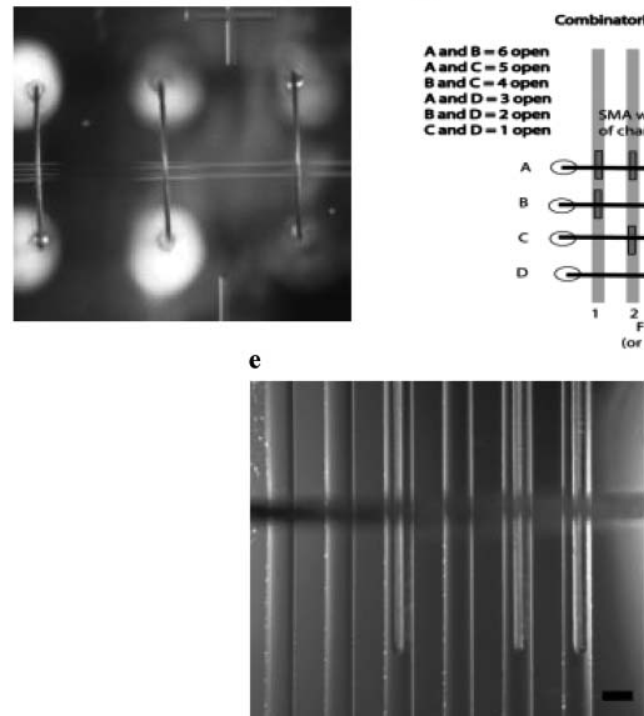

Fig. 2 Beyond single valves. (a) Block or plunger of $50 \mu \mathrm{m}$ width and $50 \mu \mathrm{m}$ height on top of a $100 \mathrm{um}$ wide channel. The circles are markers for holes into which wire is inserted. (b) One wire closing multiple channels saves space and power. (c) Valves arranged to implement a peristaltic pump. (d) A combinatorial multiplexer with plungers. The number of controlled channels scales as $2^{M}$, were $M$ is the number of wires, for large values of $M$. (e) A micrograph showing a part of a multiplexer implemented.

propose to call this a "deconvolver"-which is a combination of a multiplexer and a representation of states. Since the chip is in only one state at a given time, we operate by selecting out states one-by-one with this deconvolver. Why is a deconvolver useful? We need $\log _{2} S$ 'external' control lines to control $S$ states (Fig. 4b and Measurement and control section of Methods). Now, while the total number of states scales exponentially, the number of valid states (those needed to perform a given task) need not, and except for a multiplexer itself, where all states are used, this is the case for most fluidic chips. If the valid states scale polynomially with $N$, then for beyond some minimum number of valves, $\log _{2} S$ will always be less than $N$. Our scheme then results in considerable simplification of external control, giving a logarithmic reduction in complexity of off-chip control lines, but at the cost of extra on-chip complexity. Consider a concrete example: a chip with 10 valves. 10 valves implies $1024\left(=2^{10}\right)$ total states. If in fact the chip only requires 200 of those states to accomplish a task, we can use $\log _{2} 200$ or 8 external control lines to control all the valid states. Note that 8 external control lines is less than 10 - the number of lines needed to control each valve independently, if a deconvolver was not used. We have reduced off-chip pin-out complexity, but at the cost of greater on chip complexity. When $N$ is large, a deconvolver is advantageous; $\mathbf{a}$

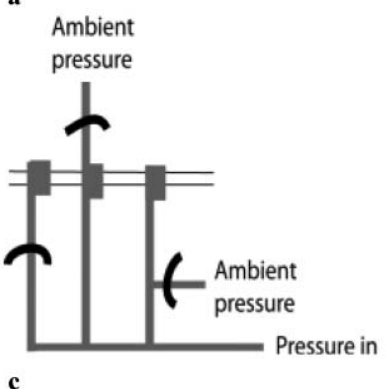

c

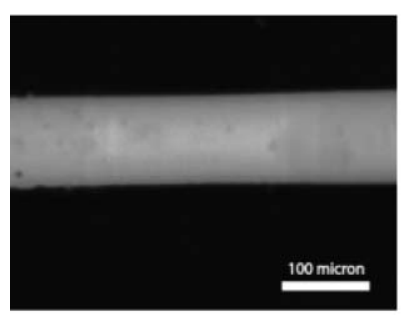

e

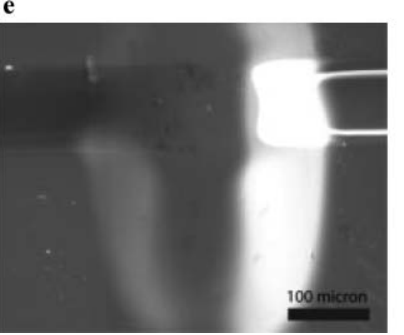

b

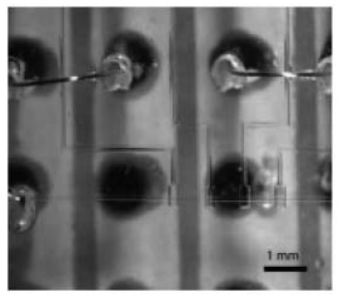

d

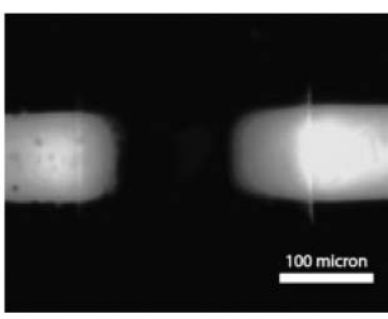

Fig. 3 Indirect valves. (a) Indirect valves can be located at various locations to create normally open or normally closed geometries. A wire upstream and another downstream of the pneumatic valve region can also be used to store pressure. (b) One of the geometries shown implemented with plunger type valves. (c) Wire is inactive resulting in pressure supply being routed to pneumatic valve. The flow channel is under 2 psi of pressure and the control channel 8 psi. The design is the same as in (b). (d) Wire is turned on causing the channel to open up. (e) A pressure latch using an ionic liquid (1-butyl-3-methylimidazolium tetrafluoroborate), instead of air. The control channel is pressurized and the wire is turned on. Then pressure is turned off. The valve remains pressurized and closed as long as the wire is electrically connected.

any chip with over a few tens of independent valves will likely benefit from it, especially when the additional cost of putting an extra valve on chip is negligible compared to the cost of an external control element. Currently, we have only implemented this scheme in software, but it may be possible to also implement it in hardware, when better automation capabilities are available. These control schemes are general, and can be implemented with other kinds of valves, besides SMA valves.

We have demonstrated that the SMA-multi-layer softlithography-PCB combination is a versatile platform, allowing us to bring to bear the entire repertoire of techniques from electronics to fluidics. While a manual procedure was used to make all valves in this paper, the process is compatible with the 'pick and place' automation used to make electronic PCBs. Moreover, the procedure of threading a string through holes with constant tension is commonly automated in industrial sewing machines. Thus, we expect that industrial scale production should not face many hurdles. 
a

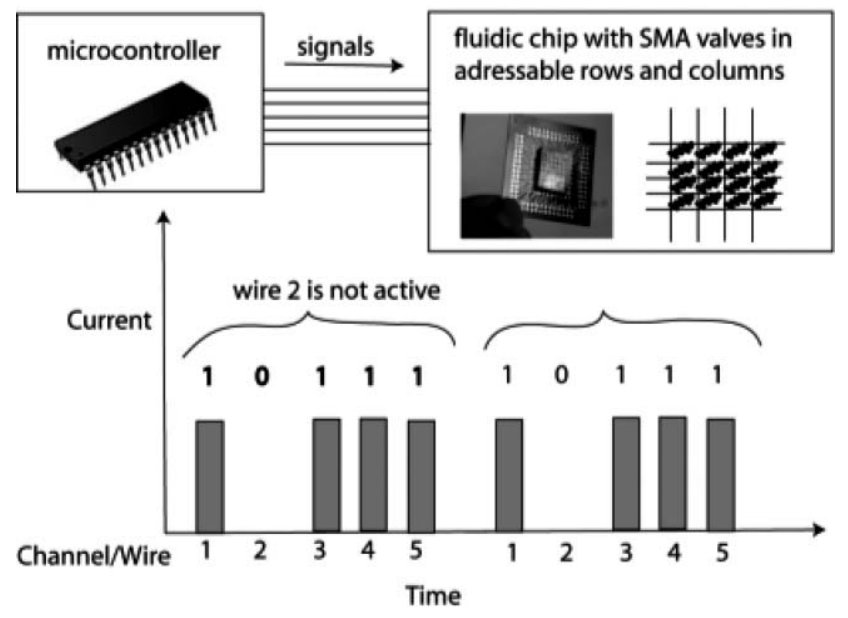

b

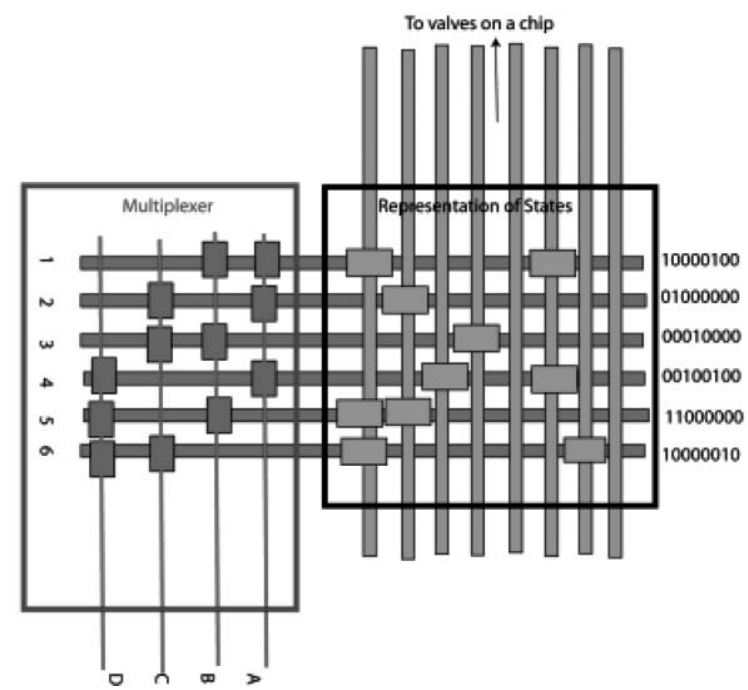

Fig. 4 Control architectures (a) Each valve is directly controlled by an external control line that carries signals from a micro-controller pin. Valves may be arranged in rows and columns to save space. Time division multiplexing, using a microcontroller allows reduction in peak power consumption as only one valve is actuated at a given time. (b) States are controlled by a deconvolver, which is a combination of the representation of states of a microfluidic chip and a multiplexer (fluidic or electronic). The multiplexer selects out states one by one. Each small rectangle represents a valve (SMA or other types) on a fluid channel or electrical line. In the figure, 4 external control lines are being used to control 6 represented states in a chip with 8 valves. Scale bar $=$ 100 micron.

By itself, making smaller fluidic circuits is not necessarily advantageous, as penalties in flow rates, and the size of biological objects (e.g. cells) limit miniaturization in an application. ${ }^{31}$ The promise here is in the control simplification of highly parallel circuits, enabling high throughput approaches with small volumes, and where analogies to electronic circuit architectures will prove most useful. We expect that an evolution of improvements in design and automation can be anticipated over time and that it will enable a new generation of portable fluidic devices with bio-medical applications. Perhaps, in the future, it will also allow us to create for biological automation, the fluidic equivalent of a microcontroller or field programmable gate array.

\section{Materials and methods}

\section{Materials}

GE RTV615 part A and B formulation was used to make the elastomeric chips. Flexinol ${ }^{\mathrm{TM}}, 3$ mil diameter wires, $70{ }^{\circ} \mathrm{C}$ transition temperature SMA wires were obtained from Dynalloy (Costa Mesa, California, USA). Solder flux was obtained from Memory-Metalle GmbH (Weil am Rhein, Germany). All other chemicals were obtained from Sigma Aldrich (St. Louis, MO, USA).

\section{Instrumentation}

Data acquisition device USB 6221 (National Instruments, Austin, TX, USA) and Keithley 2001 instrument (Cleveland, Ohio, USA) was used to measure conductivity. All other electronics was custom made. We programmed ATMEL 2561 microcontrollers (Atmel, San Jose, CA, USA) in conjunction with peripheral driver ULN2803 to actuate the wires. LM317 chips were used for adjustable voltage or current source. All electronic components were obtained from Digikey (Thief River Falls, MN, USA). Advanced Circuits (Aurora, CO, USA) made custom built PCB boards in accordance with our designs. A Nikon AZ100 stereo-microscope and was used for imaging and fluorescence.

\section{Fabrication}

The fabrication procedure for multi-layer microfluidics chips is readily available on the internet and in references. We use thermal bonding for bonding PDMS layers. After the chip is made, the remainder of the procedure involves punching holes such that they align with vias on the PCB or perforated board. The wire is inserted and soldered. It is necessary before soldering, to clean the wire free of the oxide layer that forms, and to keep the wire under some tension to prevent slack during the soldering procedure. To ensure uniform tension, we followed the following procedure: A long SMA wire is looped around and soldered to the PCB board, leaving two overhanging bits. Then a weight of $9 \mathrm{~g}$ (heat sink clip) is hung from one end. The solder is reflowed at that end, freeing the wire to be in tension to support the weight. In a few seconds the solder solidifies, preserving the wire tension. The procedure is repeated for the other end of the wire. Finally, the extra length of the wire below the PCB board is cut. The weight can be changed to create more or less tension in the wire. All the experiments in the paper used the $9 \mathrm{~g}$ weight. Finally, after soldering, the chip is washed with distilled water and dried. PDMS is poured and allowed to cure at a temperature lower than the wire transition temperature.

\section{Measurements and control}

(1) Electrical conductivity was measured by filling a channel with a solution of potassium chloride. A $50 \mathrm{~Hz}$ ac signal was applied across the channel with a $1 \mathrm{M} \Omega$ resistor in series. Voltage was monitored across the resistor and the current back-calculated. 
(2) In a combinatorial multiplexer, ${ }^{26} M$ control lines ( $M$, even) can be used to multiplex $N$ lines, with

$$
N=M ! /\left(\frac{M}{2} !\right)^{2}
$$

For large $M, N$ approaches the limit of $2^{M}$. Thus, $M$ control lines can access $2^{M}$ states.

(3) The relation between the steady state current $i_{\text {const }}$, the pulsed width modulated current $i_{\mathrm{pwm}}$ needed for actuating wires, and the duty cycle $D$ is:

$$
i_{\text {const }}=\sqrt{D} i_{\text {pwm }},
$$

Given the maximum pulsed width current, and a current needed for wire actuation in the steady state, the number of wires/ channels that can be time multiplexed is equal to $1 / D$. This assumes that the off-to-on thermal response time is smaller than the pulse rate and the off-to-on rate is larger than each time slot.

\section{Contributions}

SV suggested the initial ideas and was involved with all subsequent ideas, designs and experiments, and wrote the paper. SS, SM, DA undertook several experiments, contributed to valve design and software programming, while being mentored by SV and AS. AS contributed ideas and helped write the paper.

\section{Acknowledgements}

The authors like to thank Anna Chetverikova, Christina Morales of the Caltech Microfluidic Foundry, Ali Ghaffari of the Micro/Nano Center, Caltech for assistance in fabrication; Lyn Hein and Kate Finigan for administrative support and Glenn George for advice on electronics. All of the authors wish to thank the Boeing Company for funding this project under the SRDMA program.

\section{References}

1 G. M. Whitesides, Nature, 2006, 442, 368-373.

2 D. C. Duffy, J. C. McDonald, O. J. A. Schueller and G. M. Whitesides, Anal. Chem., 1998, 70, 4974- 4984.
3 M. A. Unger, H.-P. Chou, T. Thorsen, A. Scherer and S. R. Quake, Science, 2000, 288, 113-116.

4 T. Thorsen, S. J. Maerkl and S. R. Quake, Science, 2002, 298, 580 584.

5 W. S. N. Trimmer, Sens. Actuators, 1989, 19, 267-287.

6 S. A. Wilson, R. P. J. Jourdain, Q. Zhang, R. A. Dorey, C. R. Bowen, M. Willander, Q. U. Wahab, M. A. H. Safaa, O. Nur, E. Quandt, C. Johansson, E. Pagounis, M. Kohl, J. Matovic, B. Samel, W. Van Der Wijngaart, E. W. H. Jager, D. Carlsson, Z. Djinovic, M. Wegener, C. Moldovan, R. Iosub, E. Abad, M. Wendlandt, C. Rusu and K. Persson, Mater. Sci. Eng., R, 2007, 56, 1-129.

7 K. W. Oh and C. H. Ahn, J. Micromech. Microeng., 2006, 16, R13R39.

8 C. S. Zhang, D. Xing and Y. Y. Li, Biotechnol. Adv., 2007, 25, 483514.

9 W. Gu, X. Y. Zhu, N. Futai, B. S. Cho and S. Takayama, Proc. Natl. Acad. Sci. U. S. A., 2004, 101, 15861-15866.

10 W. Gu, H. Chen, Y. C. Tung, J. C. Meiners and S. Takayama, Appl. Phys. Lett., 2007, 90.

11 E. Smela, Adv. Mater., 2003, 15, 481-494.

12 B. Z. Yang and Q. Lin, Sens. Actuators, A, 2007, 134, 194-200.

13 R. H. Liu, J. Bonanno, J. Yang, R. Lenigk and P. Grodzinski, Sens. Actuators, B, 2004, 98, 328-336.

14 X. Z. Niu, W. J. Wen and Y. K. Lee, Appl. Phys. Lett., $2005,87$.

15 R. G. Gilbertson, Muscle Wires Project Book, Mondo-Tronics, 2005.

16 W. L. Benard, H. Kahn, A. H. Heuer and M. A. Huff, J. Microelectromech. Syst., 1998, 7, 245-251.

17 S. Shoji, Sens. Actuators, 1988, 14, 101.

18 M. E. Piccini and B. C. Towe, Sens. Actuators, A, 2006, 128, 344-349.

19 C. M. Pemble and B. C. Towe, Sens. Actuators, A, 1999, 77, 145-148.

20 C. R. Tamanaha, L. J. Whitman and R. J. Colton, J. Micromech. Microeng., 2002, 12, N7-N17.

21 D. Reynaerts, J. Peirs and H. VanBrussel, Sens. Actuators, A, 1997, 61, $455-462$.

22 F. Schiedeck, T. Hemsel and J. Wallaschek, Diffus. Defect Data, Pt. $B, 2006,113,195-198$.

23 J. P. Rolland, R. M. Van Dam, D. A. Schorzman, S. R. Quake and J. M. DeSimone, J. Am. Chem. Soc., 2004, 126, 2322-2323.

24 G. Maltezos, E. Garcia, G. Hanrahan, F. A. Gomez, S. Vyawahare, M. van Dam, Y. Chen and A. Scherer, Lab Chip, 2007, 7, 12091211.

25 W. Huang, Mater. Des., 2002, 23, 11-19.

26 Z. S. Hua, Y. M. Xia, O. Srivannavit, J. M. Rouillard, X. C. Zhou, X. L. Gao and E. Gulari, J. Micromech. Microeng., 2006, 16, 14331443.

27 P. L. Potapov and E. P. dA Silva, J. Intell. Mater. Syst. Struct., 2000, 11, 125-134.

28 M. Sreekumar, M. Singaperumal, T. Nagarajan, M. Zoppi and R. Molfino, J. Zhejiang University, 2007, 8, 818-829.

29 R. Mukherjee, T. F. Christian and R. A. Thiel, Sens. Actuators, A, 1996, 55, 185-192.

30 N. Ma and G. Song, Smart Mater. Struct., 2003, 12, 712-719.

31 K. F. Jensen, Chem. Eng. Sci., 2001, 56, 293-303. 